\title{
Stroke Care in the First Affiliated Hospital of Chengdu Medical College during the COVID-19 Outbreak
}

\author{
Li-Li Zhang ${ }^{\mathrm{a}}$ Yi-Jia Guo ${ }^{\mathrm{a}}$ Ya-Peng Lin ${ }^{\mathrm{a}}$ Ren-Zhong Hu ${ }^{\mathrm{a}}$ Jian-Ping Yu ${ }^{\mathrm{a}}$ \\ Jie Yang ${ }^{\text {a }}$ Xia Wang ${ }^{b}$ \\ aDepartment of Neurology, Clinical Medical College and The First Affiliated Hospital of Chengdu Medical College,

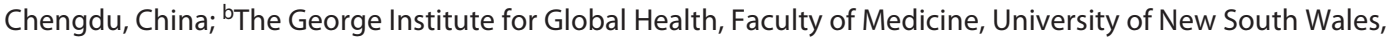 \\ Sydney, NSW, Australia
}

\section{Keywords}

Coronavirus disease-2019 · Stroke · Telestroke

\begin{abstract}
Coronavirus disease-2019 (COVID-19) has become a pandemic disease globally. The First Affiliated Hospital of Chengdu Medical College has adopted telestroke to make stroke care accessible in remote areas. During the period January 2020 to March 2020, there was no COVID-19 case reported in our stroke center. A significant reduction of stroke admission was observed between the ischemic stroke group (235 vs. 588 cases) and the intracerebral hemorrhage group (136 vs. 150 cases) when compared with the same period last year $(p<0.001)$. The mean door-to-needle time (DNT) and doorto-puncture time (DPT) was 62 and $124 \mathrm{~min}$, respectively. Compared to the same period last year, a significant change was observed in DNT (62 \pm 12 vs. $47 \pm 8 \mathrm{~min}, p=0.019)$ but not in DPT (124 \pm 58 vs. $135 \pm 23 \mathrm{~min}, p=0.682)$. A total of 46 telestroke consultations were received from network hospitals. Telestroke management in the central hospital was performed on 17 patients. Of them, $3(17.6 \%)$ patients had brain hernia and died in hospital and $8(47.1 \%)$ patients were able to ambulation at discharge and had a modified Rankin Scale of 0-2 at 3 months. The COVID-19 pandemic impacted
\end{abstract}

stroke care significantly in our hospital, including prehospital and in-hospital settings, resulting in a significant drop in acute ischemic stroke admissions and a delay in DNT. The construction of a telestroke network enabled us to extend health-care resources and make stroke care accessible in remote areas. Stroke education and public awareness should be reinforced during the COVID-19 pandemic.

ㄷ) 2020 S. Karger AG, Base

\section{Introduction}

Coronavirus disease-2019 (COVID-19) was declared a pandemic by the World Health Organization which has imposed enormous challenges to the health-care system [1]. Stroke care has been affected worldwide [2-5]. There have been many reports showing a significant decrease of stroke admissions in hospitals [6-10], as well as the delay in acute management [10-12]. The COVID-19 pandemic is changing the way we deliver stroke care [13].

Li-Li Zhang and Yi-Jia Guo contributed equally to this work and considered as joint first authors. 


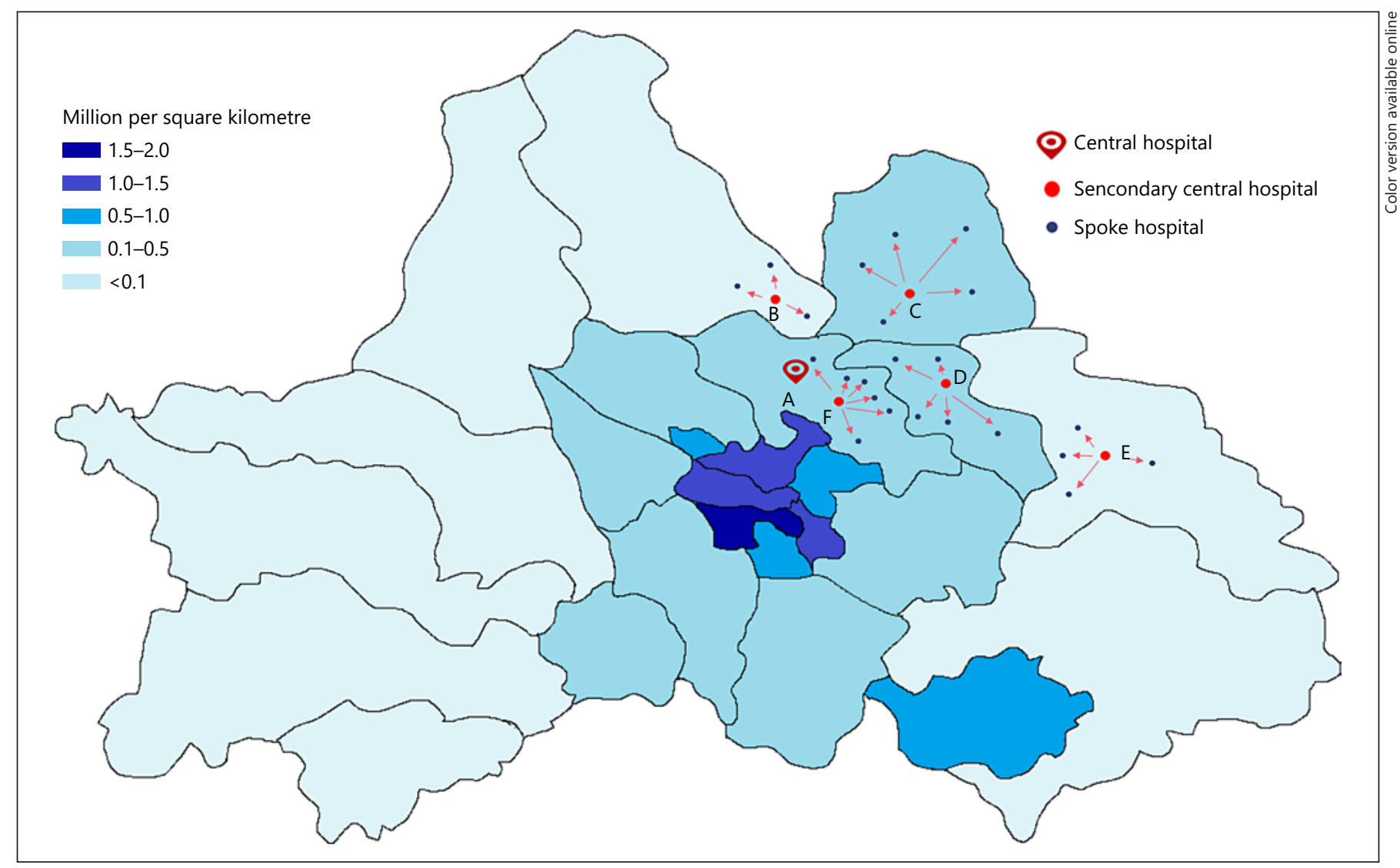

Fig. 1. Telestroke Network in Northeast Chengdu. a The First Affiliated Hospital of Chengdu Medical College. b The Second People's Hospital of Pengzhou. c Guanghan Hospital of Traditional Chinese Medicine. d Xindu Traditional Chinese Hospital Affiliated to Chengdu Medical College. e Jintang County Second People's Hospital. f The Second People's Hospital in Xindu District of Chengdu.

During the outbreak of COVID-19, The First Affiliated Hospital of Chengdu Medical College was designated as a treating hospital by the local government. This tertiary hospital was affiliated to the Health Commission of Sichuan Province, covering 4 million population in North Chengdu. It is a research and teaching hospital built in 1948, with 1,598 full-time employees and 1,500 inpatient beds. The hospital established a comprehensive stroke center affiliated to China Stroke Center Alliance in 2017. However, stroke service cannot meet the demand due to the few stroke centers in surrounding areas. Telestroke has become an increasing means to overcome the shortage of stroke expertise in recent years [14], which can improve intravenous thrombolysis (IVT) in regional areas with minor experience [15]. To provide stroke patients with timely treatment in northeast Chengdu, our hospital undertook the construction of a telestroke ser- vice to connect with regional hospitals, which was funded by Health Commission of Sichuan Province. In this study, we would like to report the stroke care in our hospital during COVID-19 pandemic.

\section{Materials and Methods}

Overall Hospital Organization in Response to COVID-19

Stroke emergency response group was set up under the leadership of COVID-19 prevention and control management team in our hospital. The director of the hospital and minister medical department served as team leader and deputy team leader, respectively. A multidisciplinary team was formed to facilitate the cooperation within the hospital. The team includes the emergency department, radiology department, laboratory department, neurology department, neurosurgery department, infectious diseases department, respiratory department, interventional treatment center, and anesthesiology department (shown in online suppl. 
Fig. 2. Stroke patients treated annually in central hospital. AIS, acute ischemic stroke; $\mathrm{ICH}$, intracerebral hemorrhage.

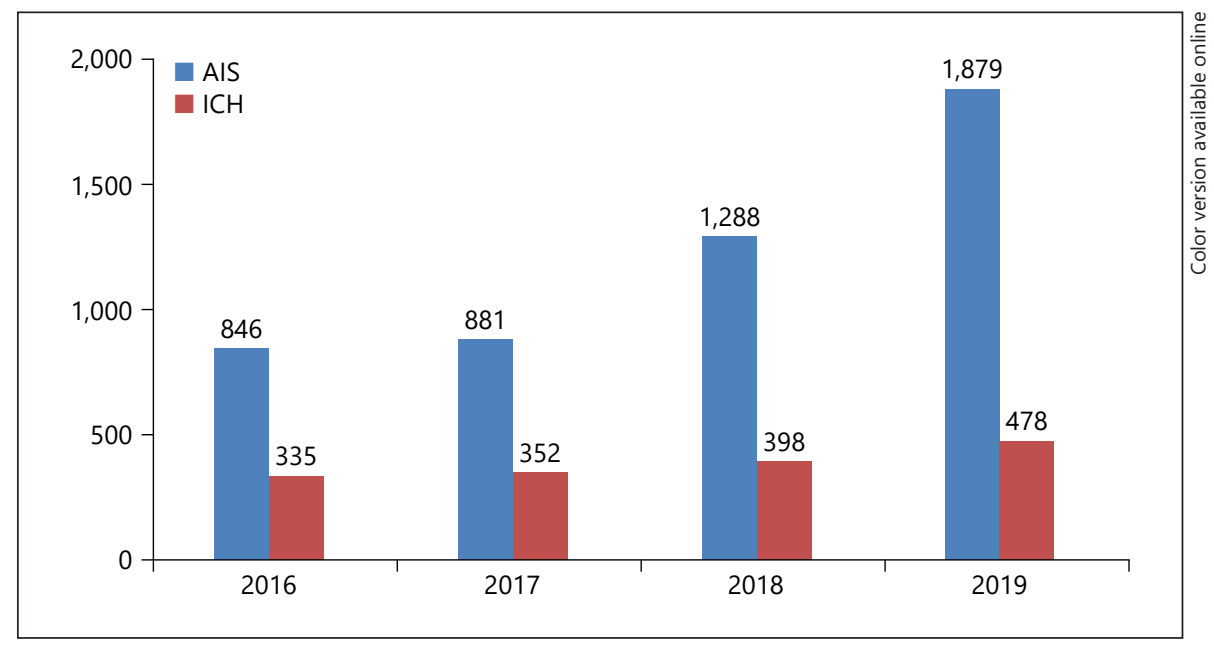

Fig. 1; for all online suppl. material, see www.karger.com/ doi/10.1159/000513097). Stroke care training, medical quality control, and regular debriefing reports were performed through teleconferences.

\section{The Construction of Telestroke Network}

Regional network in northeast Chengdu was constructed as "Hub and Spoke" Model (shown in Fig. 1). This network aimed to extend stroke care in a resource limited semi-urban area, including 3,998 square kilometer and 4 million population. Stroke network consists of 1 central hospital, 5 secondary central hospitals, and 23 spoke (primary) hospitals. We signed a cooperation agreement on tiered medical services, stroke training with secondary and primary hospitals. The infrastructure supporting the telestroke network is the telemedicine system. Consulting computers with a broadband system, video cameras, smart phones, software, and applications were set up at these hospitals. Teleconsultant data, including age, gender, symptoms, time of symptom onset, teleconsultant time, the National Institutes of Health Stroke Scale (NIHSS) score, medical history, and CT scan imaging are shared with stoke doctors. The following teleconsultant recommendations from the stroke doctors are made: (1) the patient is eligible for IVT and transfer is unnecessary; (2) the patient is eligible for IVT and must be transferred to the central hospital (for 2 main reasons, either because patients are with severe stroke or because subsequent endovascular treatment is a potential option); (3) the patient is not eligible for IVT and must be transferred to the central hospital (patients are with severe stroke or subsequent endovascular treatment is a potential option); (4) the patient is not eligible for IVT and transfer is unnecessary.

\section{Stroke Admission and Telestroke Management during}

COVID-19

A fever clinic has been incorporated into the stroke pathway to ensure patients with fever could be managed properly (shown in online suppl. Fig. 2, 3). RT-PCR testing for COVID-19 was performed to the admitted patients in all departments.

Prehospital triage during the COVID-19 pandemic provided timely transfer of stroke patients while minimizing the risk of infection exposure [16]. In our prehospital emergency system, the ambulance was equipped with a tablet computer and a smart phone, which was connected to the emergency command center in our hospital. FAST (Face, Arm, Speech, and Time) stroke scale, blood pressure, blood glucose, and heart rate were recorded in ambulance. Through transmitted video or audio and ambulance location, the stroke team could track and assess the patient in real time. During the COVID-19 pandemic, our emergency system reduced in-hospital delays at the emergency department and lowered the risk for cross-infection with other patients. However, patient delivery was not possible in some areas. Therefore, telestroke was more important than ever before to provide timely stroke care to patients, especially in remote areas. Our hospital was the central hospital as the telestroke center and the secondary central hospitals were telestroke assisted stroke units. Telestroke assisted IVT is available in secondary central hospitals. For most minor stroke patients beyond the reperfusion time window, they can receive routine treatment to avoid unnecessary transfer during the COVID-19 pandemic. For patients with severe stroke or patients within reperfusion time window, the telestroke network helps patients to be transferred to central or secondary central hospitals for IVT and to the central hospital for thrombectomy.

\section{Online Stroke Conference and Education}

Prior to the outbreak of COVID-19, academic seminars within the stroke network were face-to-face. During the pandemic, our stroke center held 2 internal coordination meetings, 2 quality control meetings, and 5 online academic conferences. Stroke patient education during hospitalization reduced significantly and was replaced by online meetings. During the COVID-19 pandemic, patients received online stroke education. Stroke patient follow-up was not affected as it was mainly performed by telephone or WeChat.

\section{Statistical Analyses}

Data were presented as actual numbers and percentages. Comparison was made using $t$ test or $\chi^{2}$ test where appropriate. All analyses were performed using Stata (V.14.0; College Station, TX, USA) and a $p$ value of $<0.05$ was considered statistically significant. 


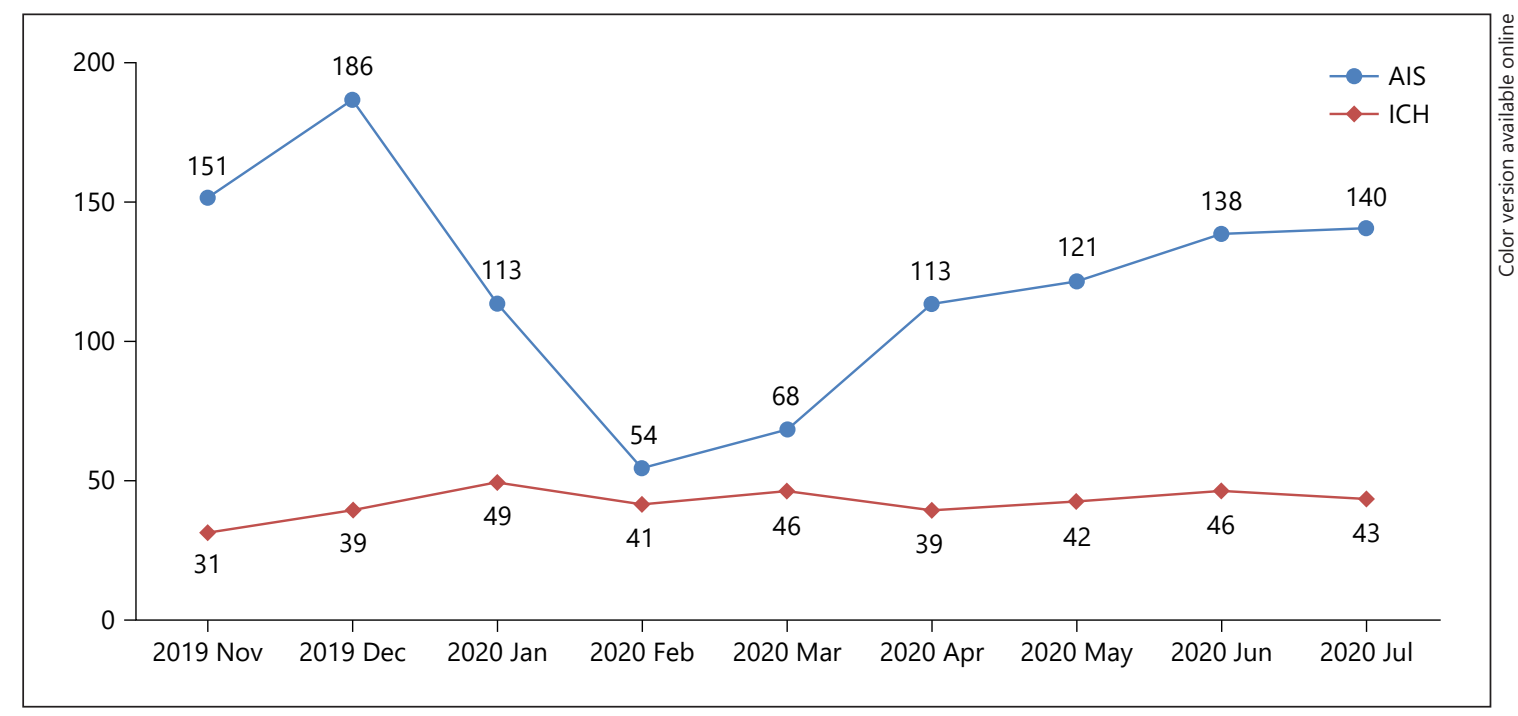

Fig. 3. Stroke admissions before and after the COVID-19 pandemic in central hospital. AIS, acute ischemic stroke; ICH, intracerebral hemorrhage; COVID-19, coronavirus disease-2019.

\section{Results}

The total number of stroke patients treated during the past years in the central hospital is shown in Figure 2. During the period January 2020 to March 2020, there was no COVID-19 case reported in our stroke center. Stroke unit staff including 6 doctors and 14 nurses were allocated to the medical team to assist COVID-19 care in Wuhan or our hospital and therefore have decreased dramatically by 20 (40\%). The number of patient admissions due to stroke had also decreased (Fig. 3). Compared with the same period last year, a significant reduction $(p<0.001)$ was observed between the acute ischemic stroke (AIS) group (235 vs. 588 cases) and intracerebral hemorrhage (ICH) group (136 vs. 150 cases).

In 235 admitted AIS patients, 20 (8.5\%) actually received reperfusion therapy. Among them, 13 patients were transferred to the central hospital for thrombectomy (4 received prior thrombolysis), 4 patients were transferred for thrombolysis, and another 3 patients receiving thrombolysis were directly contacted our stroke center. The mean door-to-needle time (DNT) and door-topuncture time (DPT) was 62 and $124 \mathrm{~min}$, respectively. Compared to the same period last year, a significant change was observed in DNT (62 \pm 12 vs. $47 \pm 8 \mathrm{~min}, p=$ $0.019)$ but not in DPT (124 \pm 58 vs. $135 \pm 23 \mathrm{~min}, p=$ 0.682).

A total of 46 telestroke consultations were received from network hospitals. Telestroke management in the central hospital was performed on 17 patients. Table 1 shows their baseline characteristic and functional outcome. Most of these patients had a moderate-severe stroke (NIHSS > 10). There were $3(17.6 \%)$ patients who had brain hernia died in hospital, 8 (47.1\%) patients were able to ambulation at discharge and had a favorable outcome (modified Rankin Scale of 0-2) at 3 months.

\section{Discussion}

This study reported stroke care in our hospital during the COVID-19 pandemic in China. We found hospital admissions related to AIS were significantly dropped. The mean DNT was 15 min longer than the last year. Moreover, we shared our preliminary experience on the construction of a telestroke network in a resource-limited semi-urban area, which may provide insight to other health institutions to provide stroke care.

For stroke admission during the COVID-19 pandemic, our findings are consistent with that from a big data analysis of 280 hospitals across China [6] and a multicenter study in Germany [7]. In prehospital setting, the admission of minor stroke has experienced the biggest drop, followed by AIS, and ICH had the smallest drop [6, 17]. This might be due to the fact that patients with minor stroke remained at home due to the fear of getting an infection if going to the hospital $[6,18]$. However, we did not observe a significant change of ICH admissions in our 
Table 1. Patient characteristic of telestroke management in central hospital

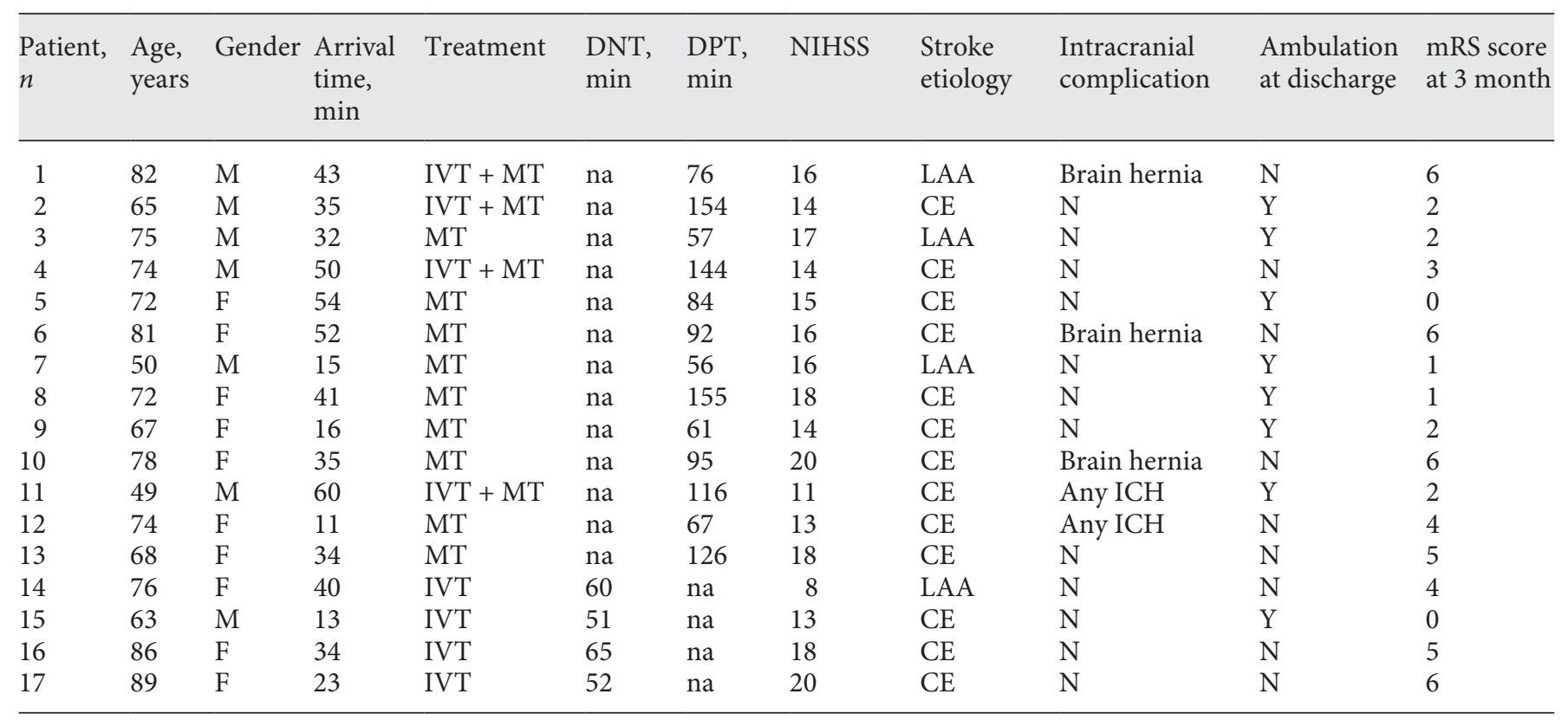

M, male; F, female; IVT, intravenous thrombolysis; MT, mechanical thrombectomy; DNT, door-to-needle time; DPT, door-topuncture time; NIHSS, National Institutes of Health Stroke Scale; LAA, large artery atherosclerosis; CE, cardioembolism; ICH, intracerebral hemorrhage; mRS, modified Rankin Scale; Y, yes; N, no; na, not applicable.

hospital, this could be partly explained by that ICH is normally a more severe type of stroke so that patients have to seek medical help rather than stay at home. In addition, the sample size for ICH is small in our report, which might be another reason to explain why our result differs from a prior study [17]. In addition, COVID-19 pandemic also impacted in-hospital stroke care; our results and the prior study [10] both observed a significant longer DNT in patients treated with IVT. The main reason for prolonged DNT was the screen procedure for COVID-19. However, this significant change was not observed in DPT for these patients with thrombectomy. This probably because the decision time for thrombectomy may be shorter when families stayed together during the COVID-19 pandemic.

This work has several limitations. First, we recognize that there were only a series of 17 patients who received telestroke care in this report. The telestroke network is more likely to capture moderate to severe patients and less likely to capture patients with minor symptoms, so we could not make any meaningful conclusion for minor stroke patients. Second, we did not collect any prehospital information, which would be helpful to understand why the number of stroke patients changed. Third, we were not able to compare the stroke care quality in our hospital before and after COVID-19 pandemic to reveal the impact of COVID-19 on stroke care.

In conclusion, the COVID-19 pandemic impacted stroke care significantly in our hospital, including prehospital and in-hospital stroke care, resulting in a significant drop in AIS admissions and a delay in DNT. The construction of a telestroke network in the northeast Chengdu region may enable us to extend health-care resources and make stroke care accessible for those who are in critical need in remote areas.

\section{Statement of Ethics}

The study was performed in accordance with the ethical principles of the Declaration of Helsinki and was approved by the Ethics Committee of The First Affiliated Hospital of Chengdu Medical College (2018CYFYHEC037). All patients signed their written informed consent to study participation.

\section{Conflict of Interest Statement}

None declared. 


\section{Funding Sources}

The First Affiliated Hospital of Chengdu Medical College is supported by a specific grant for stroke network construction from Health Commission of Sichuan Province (510000-02-092998). Dr. Guo is supported by Chengdu Medical College Science and Technology Program (CYZ19-34). Dr. Yang is supported by National Natural Science Foundation of China (81870940), Chinese Cardiovascular Association-V.G. fund (2017-CCA-VG-029), and Sichuan Science and Technology Program (2018JY0026). Dr. Wang is supported by National Heart Foundation Australia (102117) and New South Wales Health.

\section{Author Contributions}

Li-Li Zhang and Yi-Jia Guo wrote the paper. Ya-Peng Lin, RenZhong $\mathrm{Hu}$, and Jian-Ping Yu contributed to data collection. Xia Wang and Jie Yang contributed to the conception and revision of the paper.

\section{References}

1 Mahase E. COVID-19: WHO declares pandemic because of "alarming levels" of spread, severity, and inaction. BMJ. 2020;368:m1036.

2 AHA/ASA Stroke Council Leadership. Temporary emergency guidance to US stroke centers during the COVID-19 pandemic on behalf of the AHA/ASA stroke council leadership. Stroke. 2020;51(6):1910-2.

3 Aguiar de Sousa D, van der Worp HB. Stroke care in Europe during the COVID-19 pandemic. Eur J Neurol. 2020.

4 Liu L, Wang D, Brainin M, Elkind MSV, Leira E, Wang Y. Approaches to global stroke care during the COVID-19 pandemic. Stroke Vasc Neurol. 2020 Jun;5(2):107-9. https://10.1136/ svn-2020-000429.

5 Oxley TJ, Mocco J, Majidi S, Kellner CP, Shoirah $\mathrm{H}$, Singh IP, et al. Large-vessel stroke as a presenting feature of COVID-19 in the young. N Engl J Med. 2020 May 14;382(20):e60.

6 Zhao J, Li H, Kung D, Fisher M, Shen Y, Liu R. Impact of the COVID-19 epidemic on stroke care and potential solutions. Stroke. 2020;51(7):1996-2001.

7 Hoyer C, Ebert A, Huttner HB, Puetz V, Kallmünzer B, Barlinn K, et al. Acute stroke in times of the COVID-19 pandemic: a multicenter study. Stroke. 2020;51(7):2224-7.
8 Diegoli H, Magalhães PSC, Martins SCO, Moro CHC, França PHC, Safanelli J, et al. Decrease in hospital admissions for transient ischemic attack, mild, and moderate stroke during the COVID-19 era. Stroke. 2020;51(8): 2315-21.

9 Meza HT, Lambea Gil Á, Saldaña AS, Martínez-Zabaleta M, Juez PR, Martínez EL, et al. Impact of COVID-19 outbreak on ischemic stroke admissions and in-hospital mortality in North-West Spain. Int J Stroke. 2020;15(7): 755-62.

10 Montaner J, Barragán-Prieto A, Pérez-Sánchez S, Escudero-Martínez I, Moniche F, Sánchez-Miura JA, et al. Break in the stroke chain of survival due to COVID-19. Stroke. 2020; 51(8):2307-14.

11 Teo KC, Leung WCY, Wong YK, Liu RKC, Chan AHY, Choi OMY, et al. Delays in stroke onset to hospital arrival time during COVID-19. Stroke. 2020;51(7):2228-31.

12 Kerleroux B, Fabacher T, Bricout N, Moïse M, Testud B, Vingadassalom S, et al. Mechanical thrombectomy for acute ischemic stroke amid the COVID-19 outbreak: decreased activity, and increased care delays. Stroke. 2020; 51(7):2012-7.
13 Markus HS, Brainin M. COVID-19 and stroke: a global World Stroke Organization perspective. Int J Stroke. 2020;15(4):361-4.

14 Hubert GJ, Müller-Barna P, Audebert HJ. Recent advances in telestroke: a systematic review on applications in prehospital management and stroke unit treatment or telestroke networking in developing countries. Int $\mathrm{J}$ Stroke. 2014;9(8):968-73.

15 Baratloo A, Rahimpour L, Abushouk AI, Safari S, Lee CW, Abdalvand A. Effects of telestroke on thrombolysis times and outcomes: a meta-analysis. Prehosp Emerg Care. 2018 Jul-Aug;22(4):472-84.

16 Goyal M, Ospel JM, Southerland AM, Wira C, Amin-Hanjani S, Fraser JF, et al. Prehospital triage of acute stroke patients during the $\mathrm{CO}$ VID-19 pandemic. Stroke. 2020;51(7):22637.

17 Wang X, Ouyang M, Carcel C, Chen C, Sun L, Yang J. et al. Impact of COVID-2019 on stroke services in china: survey from the Chinese Stroke Association. Stroke Vasc Neurol. 2020.

18 Bersano A, Pantoni L. On being a neurologist in italy at the time of the COVID-19 outbreak. Neurology. 2020;94(21):905-6. 